\title{
Amputationen und prothetische Versorgung im Fußbereich
}

\author{
Andreas Badke, Wolfgang Gröpel, Kuno Weise
}

\section{Zusammenfassung}

Die Indikation zu einer Amputation im Fußbereich wird hauptsächlich bei ausgedehnten traumatischen Zerstörungen des Fußskelettes und der Weichteile sowie bei anderweitig nicht mehr therapierbaren Durchblutungsstörungen gestellt. Ziel der chirurgischen Behandlung ist es, so viel Standfläche wie möglich zu erhalten. Der Erhaltung der Sohlenhaut kommt bei den endbelastungsfähigen Stümpfen besondere Bedeutung zu. Durch die enormen Fortschritte in der Herstellungstechnik von Silikonprothesen ist auch bei problematischen Stümpfen eine prothetische Versorgung möglich, die hohen funktionellen und kosmetischen Ansprüchen genügt.

\section{Einleitung}

In der chirurgischen Behandlung von Erkrankungen und Verletzungen des Fußes stellt die Amputation immer die ultima ratio dar. Wenn die Zerstörung des Fußes jedoch so ausgedehnt ist, dass eine Erhaltung nicht möglich ist oder den Patienten gar gefährdet, wird eine Amputation unumgänglich. Die Indikationen, die zu einer Amputation im Fußbereich führen lassen sich in verschiedene Gruppen einteilen. Die beiden größten Gruppen stellen entsprechende Unfallfolgen und die Durchblutungsstörungen dar. Eine zahlenmäßig geringere Rolle spielen die Tumoren. Bei angeborenen Fehlbildungen sind die orthopädie-technischen Möglichkeiten so weit fortgeschritten, dass nur noch selten Amputationen erforderlich werden.

OP-JOURNAL 2001; 17: 80-83

(c) Georg Thieme Verlag Stuttgart · New York

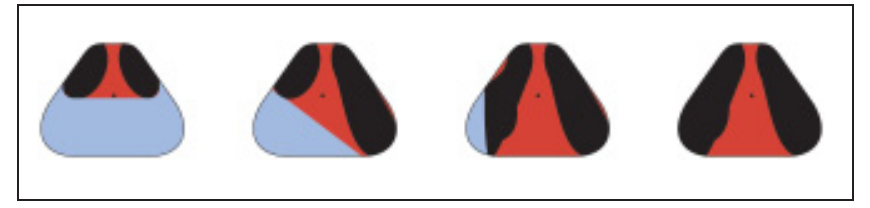

Abb.1 Standfläche nach Amputationen im Fußbereich. Der Punkt markiert das Lot vom Körperschwerpunkt. Aus (2).

Indikationen zur Amputation im Fußbereich

- Unfallfolgen

- Durchblutungsstörungen

- Infektionen

- Tumoren

- Angeborene Fehlbildungen

\section{Chirurgische Technik}

Die Wahl der Amputationshöhe wird von der Anamnese, dem klinischen Befund und ggf. apparativer Zusatzdiagnostik bestimmt. Von besonderer Bedeutung ist hierbei die Beurteilung der Hautverhältnisse, der Durchblutung, wobei sowohl der arterielle Einstrom als auch der venöse Abstrom zu beurteilen sind, die neurologische Situation (z. B. Sensibilitätsstörungen bei Polyneuropathie) und ggf. die Infektsituation. Der Fußsohlenhaut kommt hierbei besondere Bedeutung zu. Sie ist nicht nur in besonderem Maße der Belastung durch das Gehen gewachsen, ihre sensible Innervation vermittelt dem Patienten wesentliche Informationen, die für ein sicheres Gangbild von großer Bedeutung sind. Es sollte daher unbedingt versucht werden, die Sohlenhaut zu erhalten und notwendige Schnittführungen am Fußrücken zu legen.

Es sollte soviel wie möglich Sohlenhaut erhalten werden, da diese zum Einen sehr belastbar ist und zum Anderen wichtige sensible Informationen vermittelt.

Weiterhin ist bei der Wahl der Amputationshöhe zu berücksichtigen, dass sich die Standfläche durch eine Amputation im Fußbereich erheblich vermindert, so dass die Standsicherheit deutlich herabgesetzt ist (Abb.1).
Amputationen am Fußbereich sind in den in Abb. 2 dargestellten Höhen möglich.

\section{Zehenamputationen}

Die am weitesten distal gelegene Amputation im Fußbereich stellt die Grenzzonenamputation an den Zehen dar. Rechtzeitig durchgeführt kann sie bei Gefäßpatienten die Entstehung oder Ausbreitung

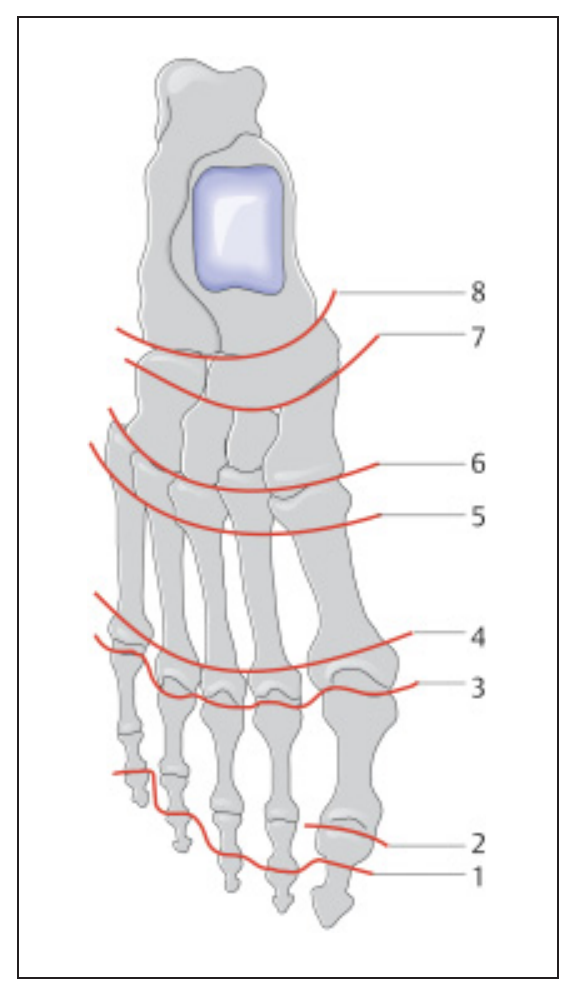

Abb.2 Mögliche Amputationshöhen im Fußbereich: Aus (2). 1 Grenzzonenamputation 2 Großzehenendgelenk 3 Zehengrundgelenke 4 Metatarsaleköpfchen 5 Basis der Metatarsale 6 Lisfranc-Gelenk 7 Bona-Jaeger-Gelenklinie 8 Chopart-Gelenk. 

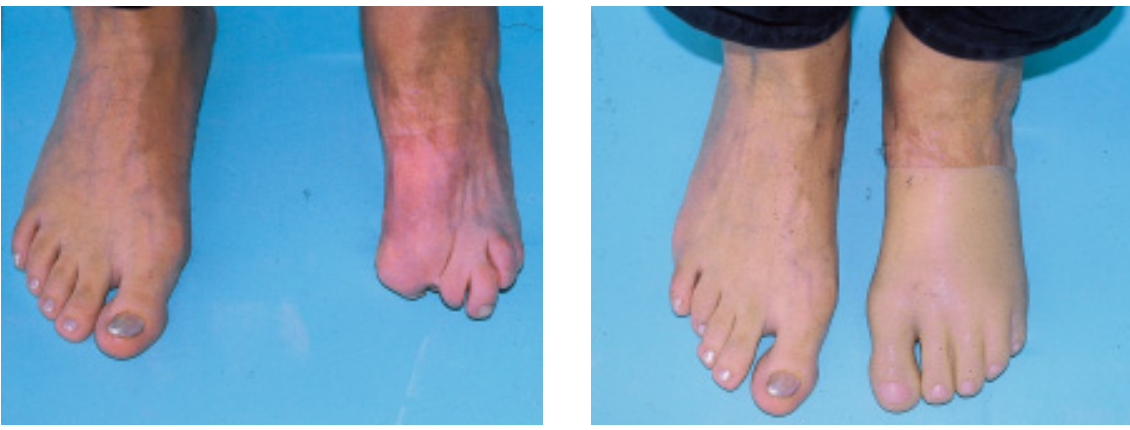

Abb. 3 Posttraumatischer Teilverlust der Zehen I, II, IV und V, prothetische Versorgung mit vorfußumgreifendem Zehenersatz.

einer Infektion verhindern. Nagel und Nagelwurzel sind immer vollständig zu entfernen. Teilamputationen der Zehen II bis $\mathrm{V}$ sind ansonsten nicht sinnvoll, da es bedingt durch das Ungleichgewicht des Muskelzugs zu Kontrakturen und neuen Druckstellen kommt. An der Großzehe kann jedoch unter Umständen eine Teilamputation sinnvoll sein. Die Zehenamputation erfolgt sonst im Sinne einer Exartikulation im Grundgelenk. Eine Resektion vitalen Gelenkknorpels des Metatarsaleköpfchens ist nicht erforderlich.

\section{Amputationen im Bereich der Metatarsalia}

Transmetatarsale Amputationen sind in verschiedenen Formen möglich und ergeben belastungsfähige und funktionell gut einsetzbare Stümpfe. Durch die Erhaltung der Ansätze der Mm. peronäus longus und brevis sowie des M. tibialis ant. bleibt das muskuläre Gleichgewicht am Fuß erhalten. Die Amputation sollte im spongiösen Bereich erfolgen, also im Bereich der Köpfchen oder der Basis. Amputationen im diaphysären Bereich führen beim Abrollen zu Schmerzen oder gar Durchspießungen der Haut. Ist die Ampu- tation einzelner Strahlen geplant muss beachtet werden, dass insbesondere die Amputation des ersten und des fünften Strahls zu einer erheblichen Beeinträchtigung der Fußstatik führt. In Einzelfällen, insbesondere beim malum perforans ist es möglich, einen oder mehrere Metatarsalia zu resezieren ohne die Zehen zu amputieren. Das Malum perforans wird hierbei chirurgisch gesäubert und kann als Drainagestelle verwendet werden.

\section{Amputationen in der Lisfranc- oder Bona-Jäger-Gelenklinie}

Eine alleinige Amputation in der LisfracGelenklinie ist bedingt durch die Asymmetrie der Fußwurzelknochen nicht sinnvoll. Die Fußwurzelknochen müssen in ihrer Länge aufeinander abgestimmt und nach plantar abgerundet werden. Hierbei müssen die direkt unter dem Knochen verlaufenden Gefäße des arcus plantaris sorgfältig geschont werden, um die Durchblutung der Sohlenhaut nicht zu gefährden. Außerdem ist eine sekundäre Verschiebung der randständigen Knochenreste nach medial und lateral ggf. durch Arthrodese mit den Nachbarknochen zu vermeiden. Bei Amputationen in der Bona-Jäger-Gelenklinie ist eine sekundäre Luxation des Navikulare nach medial und des Kuboids nach plantar zu vermeiden. Hierzu kann es sinnvoll sein, nach Resektion des Kuboids eine primäre talo-naviculäre Arthrodese durchzuführen.

\section{Exartikulation im Chopart-Gelenk}

Die 1792 erstmal beschriebene Amputation des Fußes unter Erhalt des Talus und des Kalcaneus führt im Gegensatz zu den Verfahren, bei denen der Talus geopfert wird zu keiner Beinverkürzung. Durch den Wegfall der Ansatzpunkte der Fußheber kommt es jedoch fast unvermeidbar zu einer sekundären Spitzfußstellung. Um dieser vorzubeugen wurden verschiedene Methoden beschrieben. So kombinierten Liebermann et.al. die Chopart-Amputation mit einer primären perkutanten Achillessehnenverlängerung. Zur Korrektur eines bereits eingetretenen Spitzfußes kann eine subtalare Arthrodese mit Keilresektion erforderlich werden.

\section{Kalkaneo-tibiale Arthrodese nach Pirogoff/Spitzy}

Die Pirogoff-Amputation führt zu einer Beinverkürzung um 3-4cm. Es resultiert jedoch ein voll endbelastbarer Stumpf mit erhaltener Sohlenhaut. Sie ist indiziert, wenn aufgrund der Weichteilsituation eine Chopart-Amputation nicht mehr möglich ist oder die Spitzfußstellung im oberen Sprunggelenk nicht mehr korrigierbar ist, so dass das Gelenk geopfert werden muss.

\section{Prothetische Versorgung}

Da regelrecht angelegte Fußstümpfe voll endbelastbar sind, kann der Patient auch barfuß gehen. In Abhängigkeit von
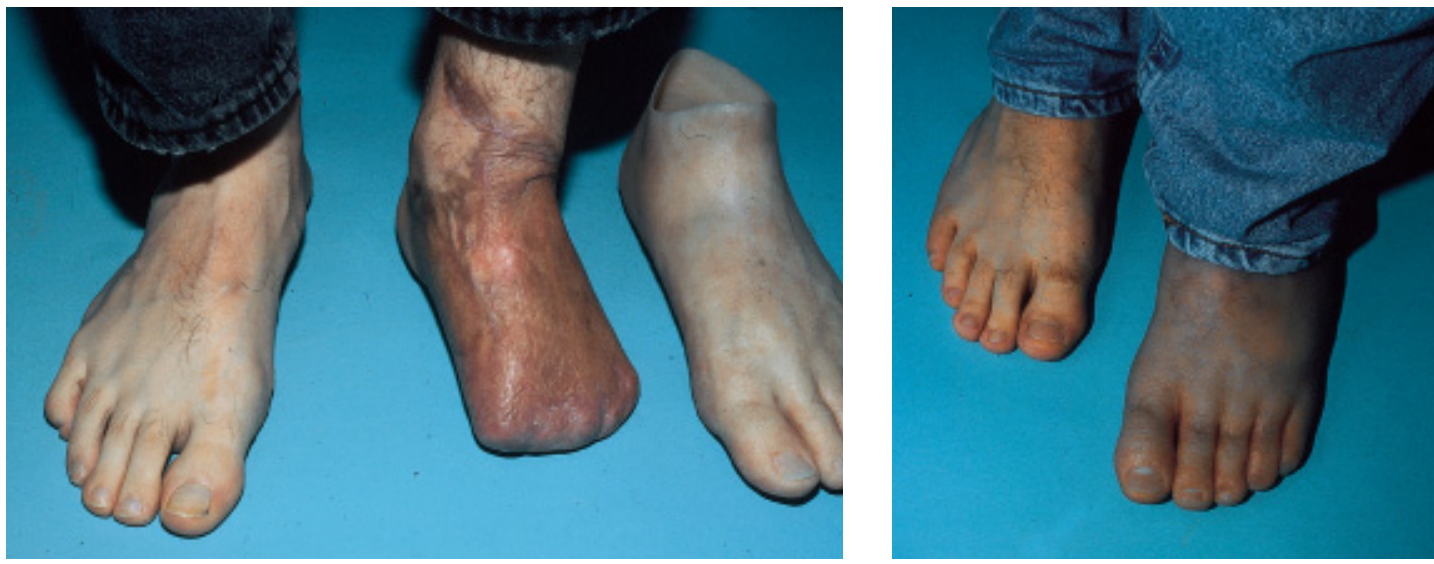

Abb. 4 Mesh-gedeckter Vorfußstumpf mit teilweise prominenten Metatarsalia, prothetische Versorgung in Silikontechnik. 
Tab. 1 Pro und Contra Silikonprothesen

\begin{tabular}{ll} 
Pro/Silikonversorgung & Contra/Silikonversorgung \\
\hline $\begin{array}{l}\text { Asthetisch ansprechend } \\
\text { sehr hygienisch (auskochbar) }\end{array}$ & Kostenaufwendig \\
wasserfest (ersetzt Badeprothese) & $\begin{array}{l}\text { Pflege aufwendig } \\
\text { spezielles know-how erforderlich } \\
\text { gutes Bodengefühl }\end{array}$ \\
$\begin{array}{l}\text { Reduzierung von Reibung am Stumpf } \\
\text { Reduzierung der Hornhautbildung }\end{array}$ \\
$\begin{array}{l}\text { freie Gelenkbeweglichkeit (OSG, USG) } \\
\text { Konfektionsschuh geeignet }\end{array}$
\end{tabular}

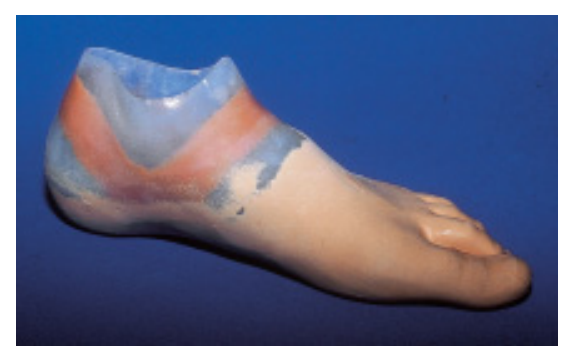

Abb.5 Probeprothese aus Silikon mit wiederverwendbarem Vorfußteil aus Pastasil.

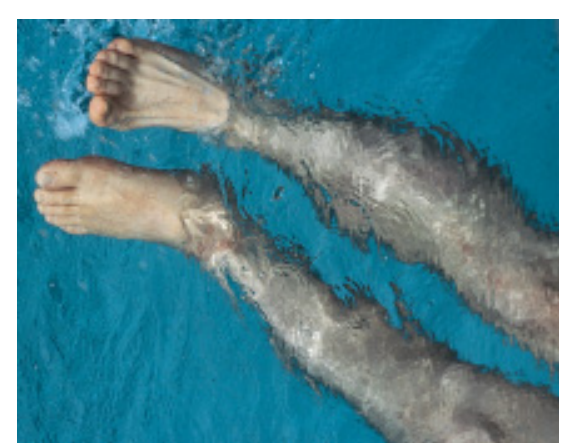

Abb. 6 Pat mit Silikonprothese im Schwimmbad.

der Amputationslinie ist es jedoch sinnvoll, die verringerte Standfläche durch prothetische Versorgung auszugleichen, wobei die verbliebenen Funktionen optimal ausgenützt werden müssen. Ebenfalls nicht zu unterschätzen sind die kosmetischen Aspekte der prothetischen Versorgung. In vielen Fällen ist für die optimale Versorgung eine enge Zusammenarbeit zwischen dem Orthopädietechniker und dem orthopädischen Schuhmacher erforderlich, da die Prothese und der Schuh als funktionelle Einheit aufzufassen sind.

\section{Zehenamputationen}

Bei Zehenamputationen wegen Durchblutungsstörungen sollte auf jede Art des Ersatzes verzichtet werden. Platzhalter können insbesondere nach Großzehenamputationen sinnvoll sein, wenn die verbliebenen in eine starke Fehlstellung abweichen, wobei insbesondere eine Rotationsabweichung den Abrollvorgang erheblich behindern kann. Bei Verlust der Großzehe kann die Verordnung einer Abrollhilfe sinnvoll sein. rigiert die Natur den verbliebenen Defekt oft durch entsprechende Stellungsänderung der anderen Zehen. Bei Amputation mehrerer Zehen kann ein kosmetischer Zehenersatz in Silikontechnik angefertigt werden. Die Fixation kann vorfußumgreifend erfolgen (Abb.3) oder erfordern, dass der Rückfuß mit eingeschlossen wird. Dies ist abhängig von der Weichteilsituation und den Anforderungen durch das Aktivitätslevel des Patienten. Bei der Prothesenverordnung ist zu berücksichtigen, dass es ein legitimes Bedürfnis des Patienten ist, im Sommer einen offenen Schuh tragen zu können.

\section{Transmetatarsale Amputationen und Amputation in der Lisfranc-Gelenklinie}

Transmetatarsale Amputationen stellen die Grenze zwischen fakultativer und obligater Prothesenversorgung dar. Bei distalen transmetatarsalen Amputationen kann eine Einlageversorgung mit Schuhzurichtung (Sohlenverstärkung, Abrollhilfe) funktionell ausreichend sein. Die gestiegenen Ansprüche der Patienten hinsichtlich der Funktion und der Kosmetik einschließlich des Tragens modischen Konfektions-Schuhwerks machen jedoch meist eine prothetische Versorgung erforderlich. Die enormen Verbesserungen in der Herstellungstechnik von Silikonprothesen zeigen hier hervorragende Ergebnisse.

Bei adhärenter Oberhaut oder großen Meshhautarealen in Belastungszonen ist eine friktionsfreie Prothesenadaption in Silikontechnik möglich (Abb.4). Verschiebeartefakte werden von der Haut weg, zwischen Prothese und Schuh geleitet.
Nach Amputationen der Zehen 2-5 kor-
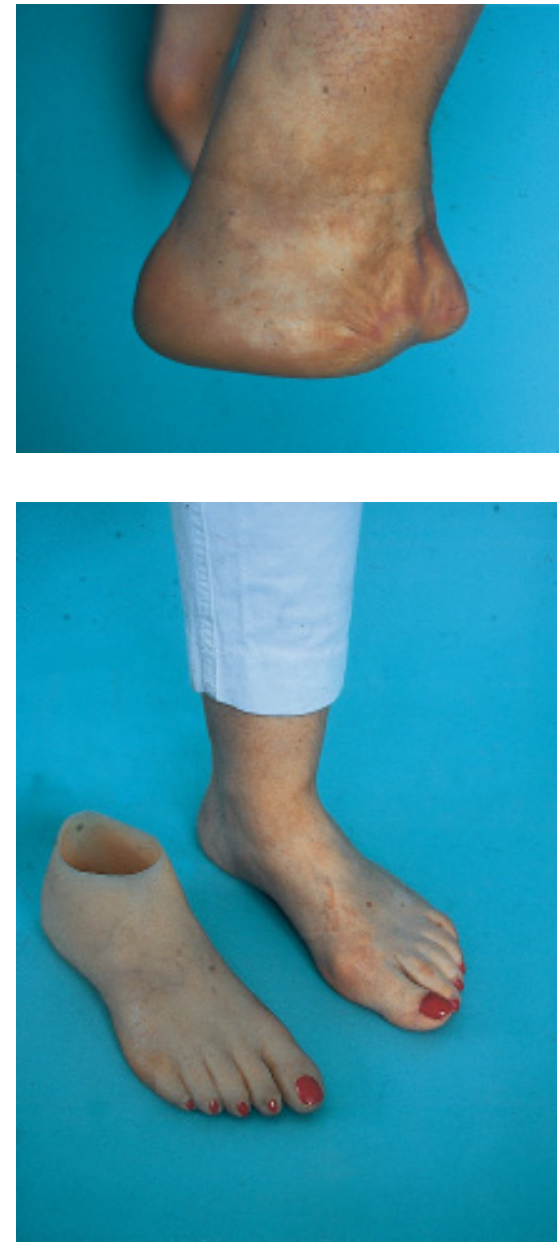

Abb. 7 Chopart-Amputation, Versorgung mit kurzer Prothese.

Durch die Herstellung einer Silikonprothese nach postoperativer Schwellung kann mittels einer Probeprothese unter Beibehaltung der Mobilität aller vorhandener Gelenke (USG, OSG) die Versorgung rasch und wirtschaftlich beginnen (Abb.5). Die Wechselmöglichkeit des Silikonschaftes unter Verwendung des in Pastasil gefertigten Vorfußersatzes ist unter ökonomischen Aspekten sinnvoll.

Empfindliche Narben oder knöcherne Prominenzen können unter Einhaltung einer Vollkontakteinbettung in sehr weichem Silikon partiell eingebettet werden. Außerdem unterstützen Silikonprothesen das chirurgische Bemühen, Sohlenhaut als propriozeptive Informationsfläche zu erhalten und auch in der Prothese zu nutzen. Die weiche Silikonsohle überträgt die Bodenreaktionskräfte dort wo sie auftreten und vermittelt dem Patienten ein natürliches Gehempfinden. Außerdem ist die Prothese wasserfest und 

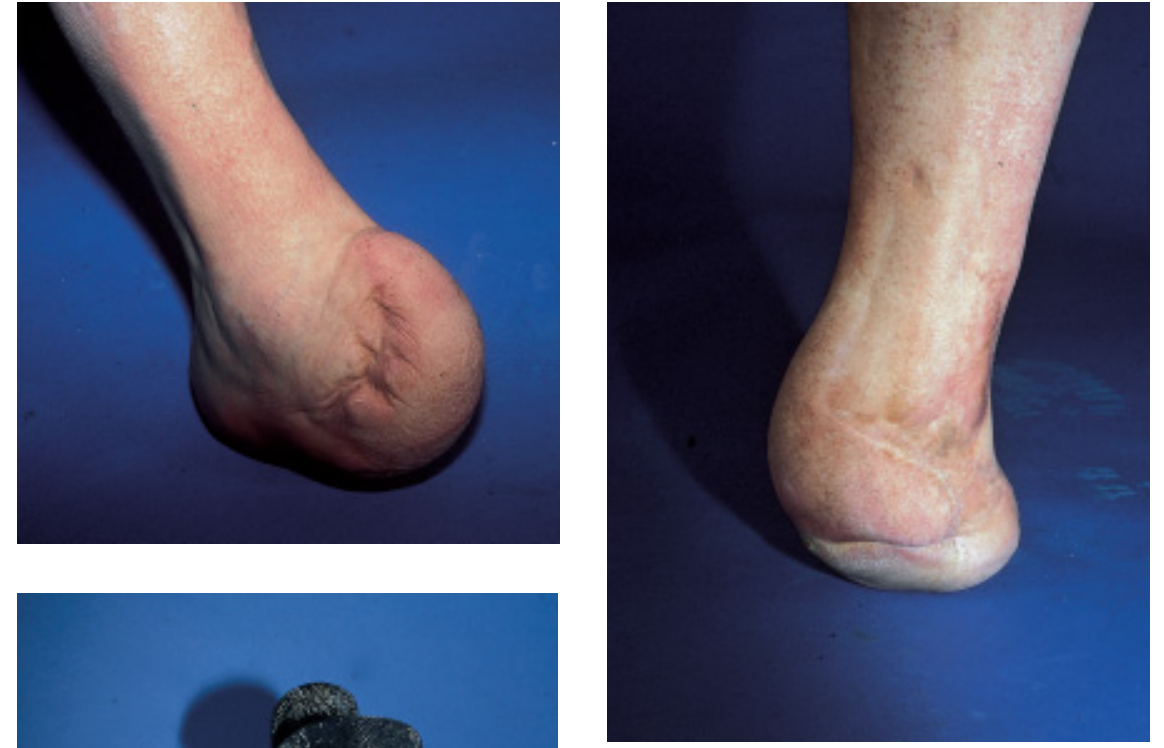

als Badeprothese im Naßbereich tragbar (Abb.6).

\section{Chopart- und Pirogof-Amputation}

Nach Chopart-Amputationen ist es bei muskelkräftigen Patienten möglich, die Beweglichkeit des oberen Sprunggelenks zumindest teilweise zu erhalten, was in einer kurzen Prothese dem Patienten wichtige Bewegungsfreiheit lässt

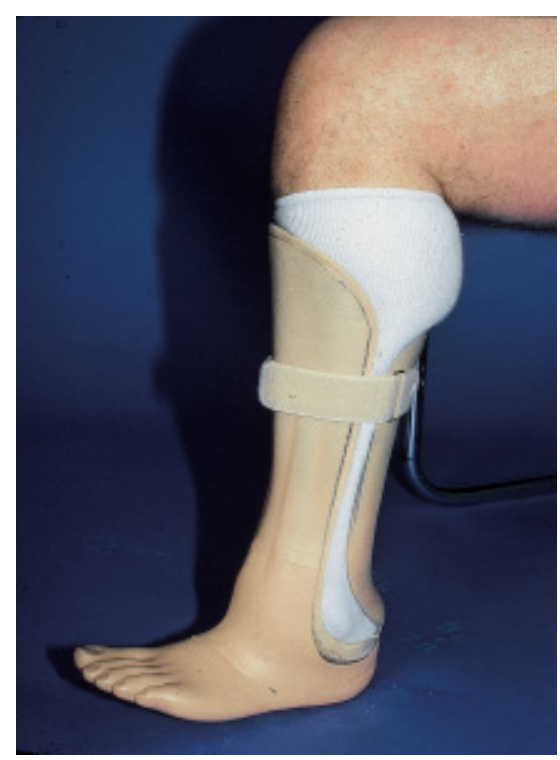

Abb.9 Pirogoff-Stumpf, Versorgung mit Prothese mit eingearbeitetem Fuß.

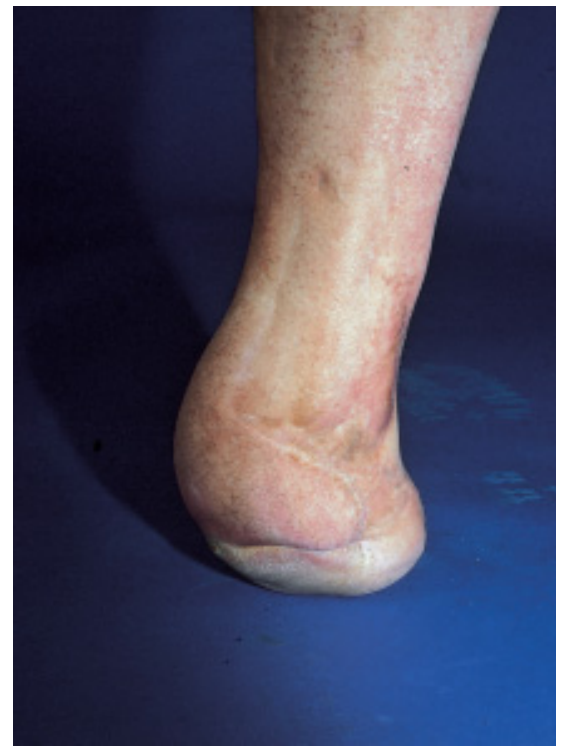

fasst und der Unterschenkel komplett in die Prothese einbezogen werden. Eine Abstützung an den Tibiakondylen zur Reduktion der Endbelastung sollte jedoch nur in Ausnahmefällen erfolgen, da dies durch venöse und lymphatische Abflussstörungen zu einer zusätzlichen Schädigung der Stumpfweichteile führen kann. Bei Chopart-Stümpfen kann es sinnvoll sein, den Rückfuß in leichter Pronation und Hackenfußstellung einzustellen.

Nach Pirogoff-Amputationen besteht eine Beinverkürzung, so dass ein Prothesenfuß in die Prothese eingearbeitet werden kann. Je nach Beanspruchung können hier verschiedene Fabrikate zur Anwendung kommen. Die Abb.9 zeigt eine Prothese für einen Pirogoff-Stumpf mit eingearbeitetem Prothesenfuß.

\section{Literatur}

1 Baumgartner R, Botta P. Amputation und Prothesenversorgung der unteren Extremität, 2. Aufl., Enke, Stuttgart 1995

2 Baumgartner R, Stinus H. Die orthopädietechnische Versorgung des Fußes, 2. Aufl. Thieme Stuttgart New York 1995

3 Lieberman JR et al. Chopart Amputation with percutaneous heel cord lengthening. Clinical orthopaedics and related research 1993; 296: 86-91

4 Taeger G, Nast-Kolb D. Amputationen und Prothesenversorgung der unteren Extremität. Unfallchirurg 2000; 103: 1097-1115

Dr. med. Andreas Badke

Oberarzt

BG-Unfallklinik

Schnarrenbergstraße 95

72076 Tübingen

Wolfgang Gröpel

Orthopädietechnikermeister

Fa. Brillinger

(Abb. 7). Ist der Stumpf zu stark weichteilgedeckt und/oder zu kurz, muss eine Rahmenprothese eingesetzt werden. Hierbei wird die Ferse eng gefasst, was dank der Carbonfasertechnik auch ohne störende umfangreiche Einbettungen zu erreichen ist (Abb.8). Die ventrale $A b-$ stützung erfolgt an der Tibiavorderkante. Bei Patienten mit instabilem OSG, schlechtem Weichteilmantel oder Sensibilitätsstörungen muss das OSG fest ge- 